\title{
SAMBAV: An Approach for Automatization of Accident Reporting System
}

\author{
Samruddhi Kulkarni ${ }^{1}$, Vaibhav Zapke ${ }^{2}$, Priyanka Jadhav ${ }^{3}$, Er. Chandan Prasad ${ }^{4}$ \\ BCA Scholar, MACT, School of Information \& Technology, Pune, India ${ }^{1}$ \\ BCA Scholar, CTIS, School of Information \& Technology, Pune, India ${ }^{2}$ \\ BCA Scholar, MACT, School of Information \& Technology, Pune, India ${ }^{3}$ \\ Assistant Professor, IT, Ajeenkya DY Patil University, Pune, India 4
}

\begin{abstract}
Security in travelling is a primary concern for everyone. Rising demand for automobile has increased the traffic, thereby causing more accidents on the road. Hence, the value of human life is ignored. No one can prevent the accident, but can save their life by expediting the ambulance to the hospital in time. This proposed work is an attempt to design and develop a smart anti-theft system that uses GPS and GSM system to prevent theft and to determine the exact location of vehicle. With the help of space navigation system GPS locates the position of the vehicle where accident has occurred. The proposed system is an automated system to provide quick assistance in case of accidents with the help of various features such as Profile Mechanism, Map View Mechanism, Alert Condition, Contact List Mechanism, Vehicle Tracking Mechanism, Emergency Button (SOS/Scream Alarm) Mechanism, Messaging Mechanism, Story Capture Mechanism, Traffic Police Intimation Mechanism, Police Intimation Mechanism, Traffic Control Mechanism, Hospital Intimation Mechanism, emergency Handling Prior Arrangement Mechanism, Ambulance at Spot Mechanism and Capture Mechanism.
\end{abstract}

Keywords: GPS, GSM, Notification, Traffic Control, Hospital Alert, Family Alert, SOS Service, SMS, Web Features, App Features, Story Capturing, Pre Diagnosis, Police Reporting, Location Tracking, Gesture Sensor, Panic Button, RTO Intimation, Social Publishing, Alarm, Call, Map, Vehicle Tracking.

\section{INTRODUCTION}

The extraordinary demand of automobiles has also increased the traffic hazards and the road accidents. Life of the people is under high risk. Now-a-days lots of accidents happen on highways due to increase in traffic and also due to rash driving of the drivers. This is because of the lack of best emergency facilities available in our country. And in many situations the family members or the ambulance and police authority is not informed in time.

This result in delaying the help reached to the person suffered due to accident. Our project Real Time Vehicle Tracking and Accident Detection with GPS is designed to avoid such situations. This design focuses on providing basic information on the accident site to the hospital or police station. As a result of this sudden help, precious life may get saved.

\section{A. GPS SYSTEM:-}

GPS modem system is stand for "Global Positioning System." The Global Positioning System (GPS) is a space based radio-navigation system consisting of a constellation of satellites and a network of ground stations used for monitoring and control. It is the receiver that collects data from the satellites and computes its location anywhere in the world based on information it gets from the satellites.

\section{B. GSM SYSTEM:-}

GSM modem system is stand for "Global system for mobile communication". A GSM modem is a specific type of modem which accepts a SIM card, and operates over a subscription to a mobile operator, just like a mobile phone. From the mobile operator outlook, a GSM modem looks just like a mobile phone.

While these GSM modems are most commonly used to provide mobile internet connectivity, many of them can also be used for sending and receiving SMS and MMS messages. In most parts of the world, GSM modems are a cost effective solution for receiving SMS messages, because the sender is paying for the message delivery. With the help of GPS and GSM module vehicle is traced.

\section{TRAFFIC CONTROL SYSTEMS:}

Whenever a traffic signal section receives the information about the accident, the RF receiver in this section is turned ON to search for ambulance close to the traffic signal. Control the traffic signal automatically with the help of RF module. Whenever the emergency vehicle reaches near to the traffic signal, the traffic signal will be made of green via $R F$ communication. Thereby the ambulance is suggested to reach the hospital without delay. 


\section{International Journal of Innovative Research in Electrical, Electronics, Instrumentation and Control Engineering}

\section{ISO 3297:2007 Certified}

Vol. 5, Issue 3, March 2017

\section{LITERATURE SURVEY}

The Prompt growth of technology and infrastructure has made our lives calmer. The advent of technology has also increased the traffic hazards and the road accidents take place regularly which causes huge loss of life and property because of the poor emergency facilities. This paper will provide a finest solution to this draw back. This paper presents vehicle accident discovery and alert system with SMS to the user defined mobile numbers. The GPS tracking and GSM alert based algorithm is designed and executed with LPC2148 MCU in embedded system domain. The proposed Vehicle accident detection system can track physical information automatically and sends an alert SMS regarding accident. Experimental work has been accepted out carefully. The result shows that higher compassion and accuracy is indeed achieved using this project. EEPROM is interfaced to store the mobile numbers permanently. This made the project more user friendly and reliable.

This paper [2] proposes a new dimension in order to allow early response and rescue of accident losses; saving lives and properties. Proposed system uses the capability of GPS and GSM along with the android phone to provide a solution which can be used to exactly detect the accident spot and to send the emergency notification to the nearby hospital's ICU and to the victim's relatives Sensors and the switches/other components used in system is dispersed throughout the car hence provides more flexibility while mounting into the vehicle. Using the open source android adds another advantage as we can work on top of some already made APIs for GPS and GSM interfacing hence decreasing the total project completion time. Overall the system performance is increased.

In highly occupied Countries [3] like India, everyday people lose their lives because of accidents and poor emergency facilities. These lives could have been saved if medical services are provided at the right time. This paper indicates system which is a solution to this problem. Accelerometer sensor can be used in car security system to sense vibrations in vehicle and GPS to give location of vehicle, so unsafe driving can be detected Automatic accident discovery and reporting system is designed in this paper. When accident occurs, it is detected by Accelerometer. Short message containing location of accident obtained using GPS, is sent via GSM network. It provides more than $70 \%$ security for four wheelers.

This paper [4] defines about a key to block a vehicle by pointing a SMS, and only a approved person unlock using security code, and helps the injured person. They used GSM and GPS modem [6] the use of microcontroller based GSM communication helps to identify the missing vehicles. GSM modem had the authorized user registered details. The intelligent traffic light controller that was announced, that saves the waiting time and avoids the traffic load. With an embedded sensor network technology, the overcrowding road is detected and managed accordingly with controllers [7]. Alarm device guess the accident vehicle using the algorithm developed. The acceleration sensors and angle sensors module delivers the necessary data to the controller. The area of accident is perceived using detection algorithm built in the controller [8].

Wireless Sensor Networks (WSN) has been applied in various fields like weather monitoring, military, home automation, health care monitoring, security and safety etc [9]. The vehicle system is placed inside the vehicle which detects the accident location by resources of sending a message. With the help of GPS and GSM module anywhere in the vehicle is traced. GSM modem used to send a precise location of the vehicle [10].

Many of the authors explicated the vehicle tracking in embedded system. One of them, Benjamin Coifman [11], explained a real-time computer vision system for vehicle tracking and traffic surveillance.R.Ramani, S. Valarmathy, Dr. N. SuthanthiraVanitha, S. Selvaraju, M. Thiruppathi, R. Thangam [12] explained Vehicle Tracking and Locking System. Their paper projected a novel method of vehicle tracking and locking systems used to track the taken vehicle by using GPS and GSM technology. KunalMaurya, Mandeep Singh, Neelu Jain [13] explained the vehicle tracking system fixed in a vehicle to enable the holder or a third party to track the vehicle's place. This design will nonstop watch a moving Vehicle and report the status of the Vehicle on demand. Chen Peijiang, Jiang Xuehua [14] explained the remote monitoring system based on SMS and GSM. System includes two parts that are the monitoring center and the remote monitoring station. The monitoring centers consist of a computer and communication module of GSM. The demo shows that the system can watch and control the remote communication between the monitoring centre and the remote monitoring station. V.Ramya, B. Palaniappan, K. Karthick [15] explained the system which provides vehicle cabin safety. This system monitors the level of the poisonous gases such as CO, LPG and alcohol within the vehicle and provides alert information as alarm during the risky situations. The system sends SMS to the authorized person through the GSM. Discovery of gases prevents further accidents. Albert Alexe, R. Ezhilarasie [16] Explained system based on cloud computing organization. In this system sensors are used to monitor the fuel level, driver conditions, and speed of the vehicle. All the data transferred to cloud server -using GSM enabled device. All the vehicles prepared with GPS antenna to locate the place. To avoid the drunk and drive, the alcohol sensor installed to monitor the driver status.

D. Taxonomy Chart:

The chart represents the analysis of various systems on various parameters to give the proposed system. Hence, 
we are able to analyse the systems which are already implanted in context of the proposed parameters which will help for realization of "SAMBAV: An Approach of Automatization for Accident Reporting System".

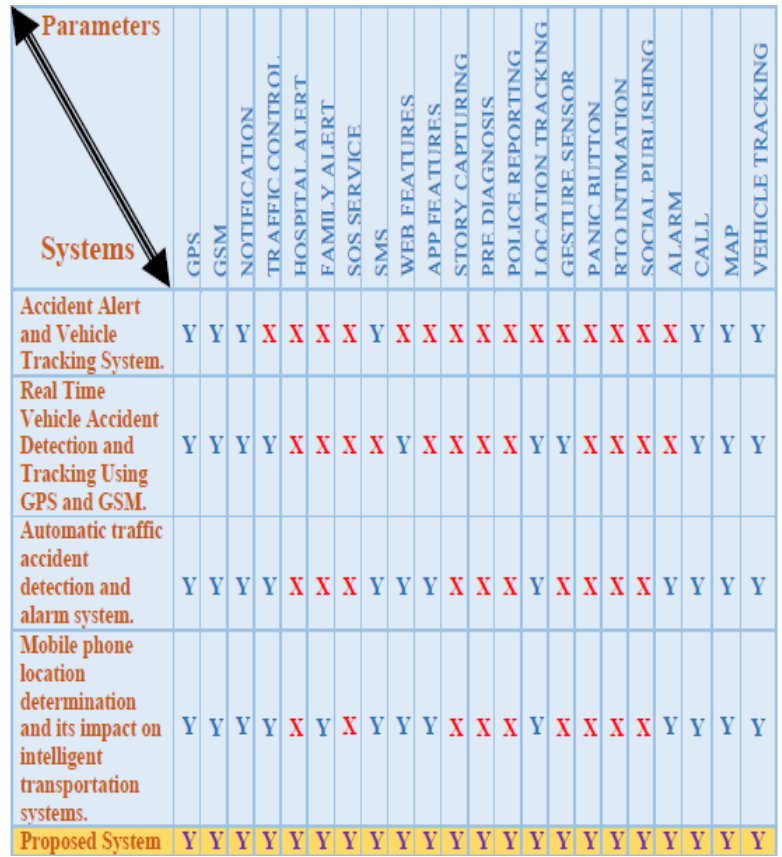

Fig 1. Taxonomy Chart

\section{III.PROPOSED SYSTEM}

The proposed system has the ability to perform various tasks (in foreground and background) to help providequick and efficient assistance in times of accident.

The system uses various mechanisms such as Profile Mechanism, Map View Mechanism, Alert Condition, Contact List Mechanism, Vehicle Tracking Mechanism, Emergency Button(SOS/Scream Alarm) Mechanism, Messaging Mechanism, Story Capture Mechanism, Traffic Police Intimation Mechanism, Police Intimation Mechanism, Traffic Control Mechanism, Hospital Intimation Mechanism, emergency Handling Prior Arrangement Mechanism, Ambulance at Spot Mechanism and Capture Mechanism.

In the proposed system the user details will be synced with the help of user's social media accounts (e.g. Facebook, LinkedIn, etc.). By this user can view their location on map via geocoding. A message is sent to the contact list of the user. It creates an SOS message or Scream Alarm in case of no network areas. We can track the vehicle with the help of embedded device in the vehicle. In case of accident the nearest Traffic Police, Police Station and Hospital will be intimated. An ambulance having all the facilities required at the accident location will be summoned. The user can post on social media and also get help from that platform.

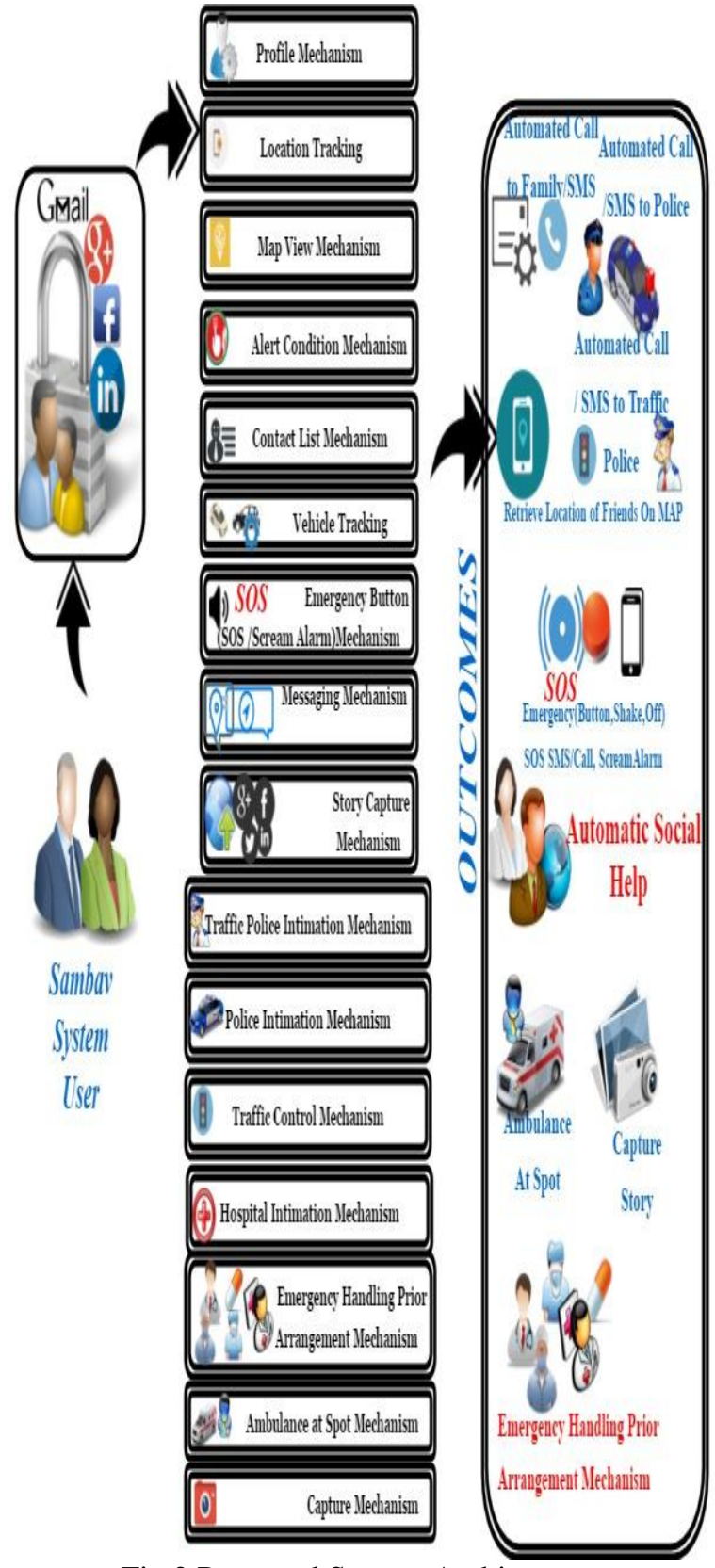

Fig.2 Proposed System Architecture.

\section{IV.CONCLUSION}

This is "SAMBAV: An Approach for Automatization of Accident Reporting System" which will prove very useful for victims in accident in deserted areas. Along with the App we will embed a device in the vehicle which will help send location of the victim to the nearest police stations, traffic police and hospitals. The proposed system is more secure, smart and safe application in all the context for providing assistance for those in need. Other features include sending notification to the victim's family and uploading image \& status on social media. This app will prove very useful in reducing the death rate due to accidents. 
Vol. 5, Issue 3, March 2017

\section{REFERENCES}

[1] Muhammad Ali Mazidi and Janice GillispieMazidi, THE 8051 MICROCONTROLLER AND EMBEDDED SYSTEMS, Pearson Education.

[2] C.Prabha, R.Sunitha, R.Anitha, AUTOMATIC VEHICLE ACCIDENT DETECTION AND MESSAGING SYSTEM USING GSM AND GPS MODEM,IJAREEIE 7, July 2014.

[3] Vikram Singh Kushwaha, Deepa Yadav, CAR ACCIDENT DETECTION SYSTEM USING GPS, GSM AND BLUETOOTH, IJERGS May-June 2015.

[4] AboliRavindraWakure,ApurvaRajendraPatkar,VEHICLE ACCIDENT DETECTION AND REPORTING SYSTEM USING GPS AND GSM, IJERGS April 2014.

[5] Tang S Huming, Gong Xiaoyan, Wang Feiyue, Senior Member, Traffic Incident Detection Algorithm based on Non-Parameter Regression, IEEE, 2002, pp7389-7803.

[6] Hu Rufua, Li Chuanzhia, He Jieb, Hang Wenb and Tao Xianglib, Study on the Method of Freeway Incident Detection using Wireless Positioning Terminal ICICTA in Hunan, IEEE, 2008, pp695-978.

[7] Rajesh Kannan Megalingam. Ramesh Nammily Nair, Sai ManojPrakhya, Wireless Vehicular Accident Detection and Reporting System, IEEE, 2010.

[8] Md. Syedul Amin, JubayerJalil, M. B. I. Reaz, Accident detection and reporting system using GPS, GPRS and GSM technology, IEEE, 2012.

[9] A. Dardanelli, F. Maggi, M. Tanelli, S.M. Savaresi, R. Kochanek and T. Holz, A Security Layer for Smartphone-To-Vehicle Communication over GSM, IEEE Embedded Systems Lett, vol. 5, 2013, pp 34-37.

[10] YogitaJadhav and Ganesh Wayal, A survey-security layer for smartphone -to-vehicle communication over GSM, International Journal of Engineering and Techniques - Volume 2 Issue 3, May June 2016.

[11] Kiran Sawant,ImranBhole,PrashantKokane, Pirajidoiphode, Prof. Yogesh Thorat, Accident Alert and Vehicle Tracking System, ISSN 2348120X Vol. 3, Issue 4, pp: (259-263), Month: October December 2015, Available at: www.researchpublish.com.

[12] Benjamin Coifman, A real-time computer vision system for vehicle tracking and traffic surveillance, Transportation Research Part, 2003.

[13] R. Ramani, S. Valarmathy, Dr. N. SuthanthiraVanitha, S. Selvaraju, M. Thiruppathi, R. Thangam, Vehicle Tracking and Locking System Based on GSM and GPS. I.J. Intelligent Systems and Applications, 2013, 09.

[14] KunalMaurya, Mandeep Singh, Neelu Jain, "Real Time Vehicle Tracking System using GSM and GPS Technology- an Anti-theft Tracking System," International Journal of Electronics and Computer Science Engineering. ISSN 2277-1956/V1N3-11031107.

[15] V.Ramya, B. Palaniappan, K. Karthick, Embedded Controller for Vehicle In-Front Obstacle Detection and Cabin Safety Alert System, International Journal of Computer Science \& Information Technology (IJCSIT) Vol 4, No 2, April 2012

[16] Albert Alexe, R. Ezhilarasie, Cloud Computing Based Vehicle Tracking Information Systems, ISSN: 2229 - 4333 (Print) | ISSN: 0976 - 8491 (Online) IJCST Vol. 2, Issue 1, March 2011. 